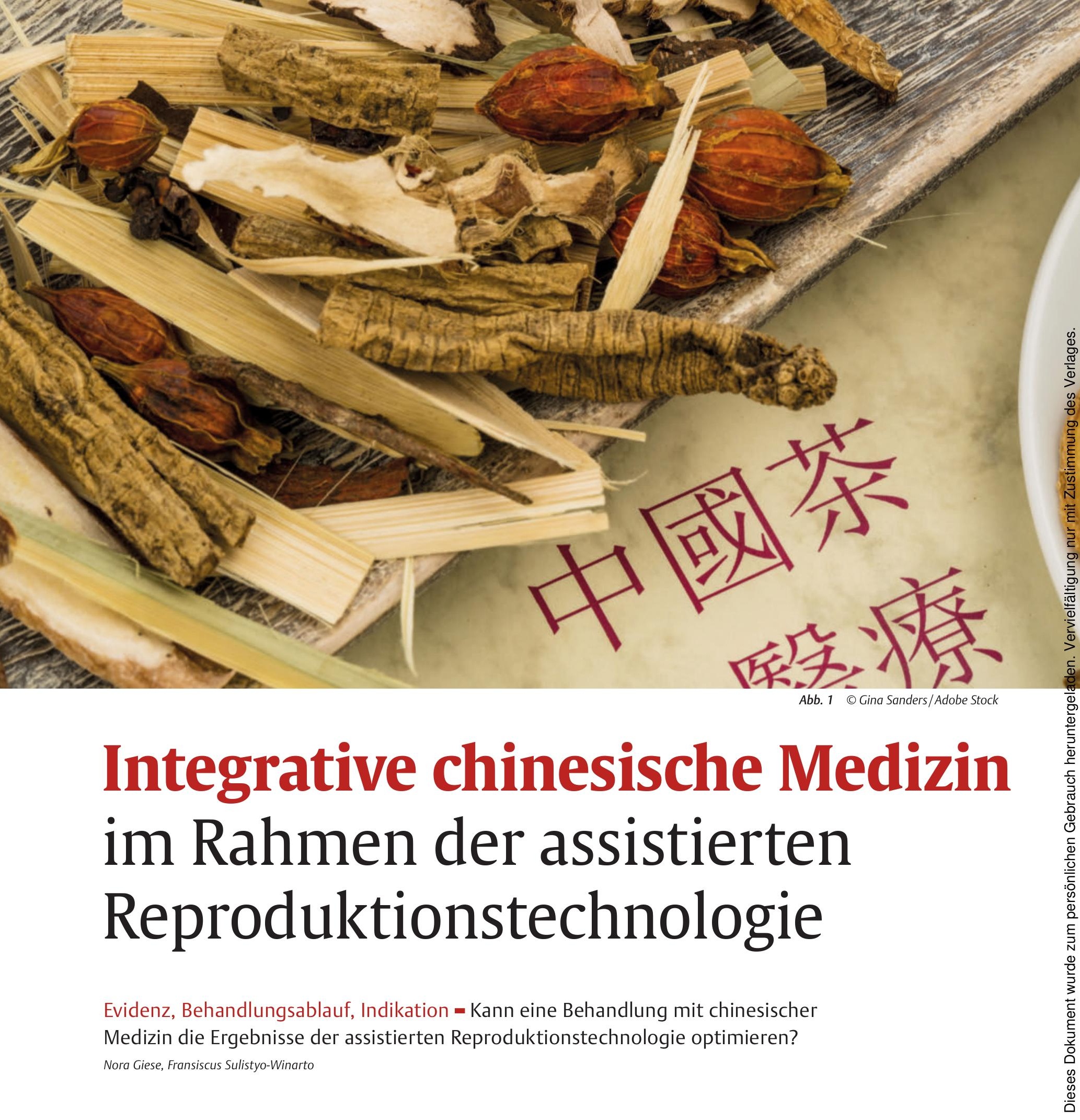

Um ihre Chancen auf eine Schwangerschaft zu erhöhen, suchen Paare zunehmend Unterstützung in der Reproduktionsmedizin. 3\% aller Kinder, die 2015 in Deutschland geboren wurden, entstanden mithilfe der assistierten Reproduktionstechnologie (ART). Im Jahr 2016 wurden in Deutschland 103981 Embryonen nach einer künstlichen Befruchtung - In-vitro-Fertilisation (IVF) oder intrazytoplasmatischen Spermieninjektion (ICSI) - transferiert, also in den Uterus zurückgegeben. Diese Zahl hat sich seit 2004 nahezu verdoppelt [2].

Trotz dieser großen Erfolgsgeschichte stagnieren die klinischen Schwangerschaftsraten nach ART bei etwa $30 \%$ pro Transfer [2]. Die- se Zahlen sind für alle Beteiligten nicht komplett zufriedenstellend und so suchen Patientinnen und Reproduktionsmediziner nach weitergehenden Behandlungsoptionen. Zusätzlich zu den Maßnahmen der assistierten Reproduktionsmedizin sehen betroffene Frauen einen integrativen Behandlungsansatz mit chinesischer Medizin (CM) als sinnvoll an [9].

Die CM wird seit mehr als 2000 Jahren eingesetzt, um gynäkologische Erkrankungen und einen unerfüllten Kinderwunsch zu behandeln. Heute wird sie zunehmend integrativ eingesetzt. Von einem integrativen Behandlungsansatz spricht man, wenn die Therapie mit CM in Abstimmung auf die Maßnahmen der westlichen 


\section{Verwendete Abkürzungen}

ART: assistierte Reproduktionstechnologie

CAT: chinesische Arzneimitteltherapie

CM: chinesische Medizin

$\mathrm{RCT}$ : (randomized controlled trial) randomisierte,

kontrollierte Studie
Medizin auf wissenschaftlicher Basis erfolgt [5]. Unsere Fragestellung lautet: Kann eine Behandlung mit CM dazu beitragen, die Ergebnisse der assistierten Reproduktionsmedizin zu verbessern? Wie ist die Evidenz für die Effektivität von chinesischer Medizin im Rahmen der ART? Und wie kann eine solche Behandlung in der klinischen Praxis konkret aussehen?

\section{Studienlage}

Die Evidenz für die Effektivität der CM im Rahmen der ART lässt sich in drei Gruppen zusammenfassen.

Die erste Gruppe untersucht die Wirksamkeit der Chinesischen Arzneimitteltherapie (CAT) im Rahmen der ART.

Die zweite und größte Gruppe befasst sich mit der Fragestellung, inwieweit Akupunktur um den Embryotransfer die Schwangerschaftsraten verbessert. Für beide Gruppen existieren systematische Übersichtsarbeiten, die die Ergebnisse zusammenfassen.

Die dritte - sehr kleine - Gruppe umfasst einzelne Studien, die die Wirksamkeit der CM als Medizinsystem untersuchen, bei der verschiedene Behandlungsformen wie Akupunktur und CAT gemeinsam angewendet werden. Diese Addition verschiedener therapeutischer Interventionen entspricht in den meisten Fällen der Anwendung der CM im Westen, wie sie von Therapeuten praktiziert wird, die sich auf die integrative Kinderwunschbehandlung spezialisiert haben. Die Studien dieser Gruppe haben den Nachteil, durch die individualisierten therapeutischen Interventionen in der Evidenz-Hierarchie weiter unten zu stehen, allerdings kommen die Ergebnisse den tatsächlichen Behandlungsergebnissen in der klinischen Praxis näher als einzelne, meist standardisierte Verfahren, wie sie in den beiden anderen Gruppen untersucht werden.

\section{Zusammenfassung}

Ergebnisse aus aktuellen Studien legen nahe, dass die chinesische Medizin die assistierte Reproduktionstechnologie sinnvoll unterstützen kann. Aktuelle Studienergebnisse zeigen u. a. die Verbesserung der Schwangerschaftsrate bei zusätzlicher chinesischer Arzneimitteltherapie. In verschiedenen RCTs konnten Effekte der Akupunktur nachgewiesen werden, u. a. verbesserter Blutfluss zum Uterus und zu den Ovarien sowie die Entlastung von emotionalem Disstress.

Die Autoren diskutieren aktuelle Studienergebnisse und geben Empfehlungen zum Vorgehen in der Praxis sowie zu ausgewählten Rezepturen und Behandlungsschemata zur Unterstützung der assistierten Reproduktionstechnologie.

\section{Chinesische Arzneimitteltherapie und assistierte Reproduktionstechnologie}

Eine systematische Übersichtsarbeit und Metaanalyse von 2013 untersucht den Effekt von CAT im Rahmen der ART im Vergleich zu ART allein [4]. 20 randomisierte kontrollierte Studien (randomized controlled trials, RCTs) mit insgesamt 1721 Teilnehmerinnen wurden eingeschlossen. Drei davon wurden mit einem unklaren Risiko für eine Verzerrung bewertet, alle anderen mit einem hohen Risiko für Bias. Als Hauptursachen der Infertilität wurden Tubenverschluss, polyzystisches Ovarialsyndrom und Endometriose angegeben. Der Behandlungsbeginn variierte zwischen den Studien von drei Monaten vor Stimulationsbeginn bis nach dem Embryotransfer. Verschiedene Standardrezepturen wurden eingesetzt; keine Studie berichtet über eine individualisierte Anpassung der Verschreibung entsprechend der Symptome und Zeichen, wie sie in der täglichen Praxis üblich ist. Die Metaanalyse zeigt eine signifikante Verbesserung der Schwangerschaftsraten von 36,2\% in den Kontrollgruppen auf $53,2 \%$ in den Interventionsgruppen. Die Kombination von CAT und ART verbessert die klinischen Schwangerschaftsraten $(p<0,00001)$ und andauernden Schwangerschaftsraten $(p=0,009)$ signifikant.

Ergänzend sei an dieser Stelle eine Metaanalyse von 2015 erwähnt, die den Effekt individualisierter Verschreibungen der CAT auf die Fertilität untersucht, die allein oder integrativ mit westlicher Medizin, entweder einer hormonellen Behandlung - meist Clomifen oder Operationen angewendet wird [14]. Ausgeschlossen wurden Studien, in denen CAT im Rahmen der ART angewendet wurde. Eingeschlossen wurden 40 Studien mit insgesamt 4247 Teilnehmerinnen. Das Ergebnis zeigt eine zweifach erhöhte Verbesserung der kumulativen Schwangerschaftsraten von 33\% in den Kontrollgruppen auf $60 \%$ in den Interventionsgruppen nach drei bis sechs Monaten Behandlung mit CAT. Obwohl in dieser Metaanalyse nicht der Effekt von CAT im Rahmen der ART untersucht wurde, sind die Ergebnisse dennoch in unserem Zusammenhang interessant, da sie darauf hinweisen, dass eine längere Behandlungsdauer sowie individualisierte Verschreibungen - im Vergleich zu standardisierten Verschreibungen die Schwangerschaftsraten zusätzlich verbessern. Aufgrund eingeschränkter Informationen zur Methodologie wurde das Risiko für Bias auch in dieser Metaanalyse als hoch eingeschätzt.

\section{Akupunktur und assistierte Reproduktions- technologie}

Eine ganze Reihe von RCTs untersucht die Effektivität von Akupunktur im Rahmen der ART. Verschiedene Mechanismen konnten identifiziert werden, die das Ergebnis beeinflussen: Akupunktur verändert neuroendokrinologische Faktoren und die Immunfunktion, verbessert den Blutfluss zum Uterus und zu den Ovarien, hemmt die uterine Motilität und entlastet emotionalen Disstress ebenso wie Depression und Angst/Sorge [1, 7].

Eine der ersten RCTs zur Effektivität der Akupunktur zur Verbesserung der ART-Ergebnisse wurde 2002 von Paulus et al. durchgeführt [11]. 80 Frauen wurden vor und nach dem Embryotransfer akupunktiert. Das Ergebnis zeigte eine signifikante Verbesserung der Schwangerschaftsrate von $42,5 \%$ in der Akupunkturgruppe im Vergleich zu 26,3\% in der Kontrollgruppe ( $p=0,03$ ).

Eine Reihe folgender Studien versuchte, diese Ergebnisse zu wiederholen - mit heterogenen Ergebnissen. In Anlehnung an die Pau- 


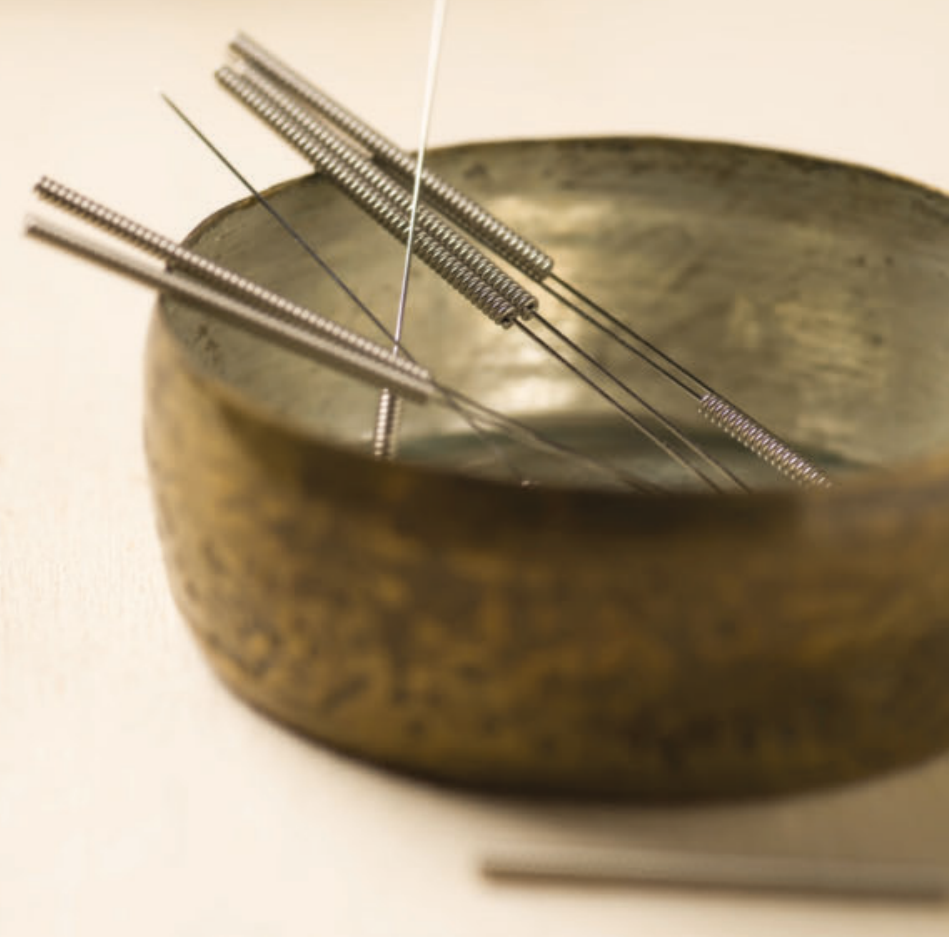

Abb. 2 (c) xiquence/Adobe Stock

\section{Die Datenlage zeigt einen deutlichen} Trend zur Verbesserung der Schwangerschaftsraten und der Lebendge-

\section{burtenraten bei integrativer Therapie mit chinesischer Medizin.}

lus-Studie wurden in den meisten dieser Studien zwei bis drei Akupunktursitzungen um die Zeit des Embryotransfers oder der Eizellentnahme angewendet. Vier systematische Überblicksarbeiten und Metaanalysen zeigten keinen Effekt von Akupunktur [6, 13, 10, 16], während eine Arbeit eine statistisch signifikante Verbesserung der klinischen Schwangerschaftsraten nach Akupunktur zeigte [18]. Die jüngste systematische Überblicksarbeit schließt 30 Studien mit insgesamt 6344 Frauen ein [12] und zeigt eine signifikante Verbesserung der Schwangerschaftsraten zwischen Akupunktur- und Kontrollgruppen $(p=0,01)$. Die Ergebnisse deuten darauf hin, dass die Schwangerschaftsraten besser ausfallen, wenn Elektroakupunktur im Vergleich zu traditioneller Akupunktur angewendet wird und deutlich besser sind, wenn Akupunktur nicht nur um den Transfer gegeben wird, sondern bereits im Vorfeld. Dieses Ergebnis stimmt mit unseren Erfahrungen aus der klinischen Praxis und der Metaanalyse von Karin Ried [14] überein, dass eine längere Behandlungsdauer zu einer Verbesserung der Ergebnisse führt. Eine naheliegen- de Erklärung für die Heterogenität der Akupunkturstudien könnte sein, dass die Oozyten- und Embryoqualität ausschlaggebend ist für den Erfolg der ART. In der Paulus-Studie wurden nur Frauen mit guter Embryoqualität zugelassen - ein Ausschlusskriterium, das in den Folgestudien nicht erwähnt wird. Die Akupunkturbehandlung um den Transfer kann also eingesetzt werden, um die Nidations- und Implantationsraten guter Embryonen zu verbessern. Kommen andere Probleme wie eine reduzierte ovarielle Kapazität oder Einschränkungen der Eizellqualität hinzu, ist eine längere und vorbereitende Behandlung mit $\mathrm{CM}$ sinnvoll.

\section{Integrative chinesische Medizin und assistierte Reproduktionstechnologie}

In der klinischen Praxis wird die CM zur integrativen Kinderwunschbehandlung in der Regel - zumindest im Westen und von auf Kinderwunsch spezialisierten Therapeuten - individualisiert angewendet. Sie umfasst nicht nur Akupunktur oder CAT allein, sondern beide Anwendungen gemeinsam sowie Empfehlungen zur Ernährung und Lebensführung. Eine amerikanische retrospektive Kohortenstudie mit insgesamt 1231 Teilnehmerinnen untersuchte die Effektivität der CM als Gesamtanwendung (Whole-Systems Traditional Chinese Medicine, WS-TCM) in Kombination mit ART im Vergleich zur Standardbehandlung mit ART allein und im Vergleich zu einer Akupunkturgruppe, in der die Teilnehmerinnen zwei standardisierte Akupunkturbehandlungen am Tag des Embryotransfers bekamen [8]. Die durchschnittliche Anzahl an Behandlungen in der WS-TCM-Gruppe betrug 12 Behandlungen über einen Zeitraum von etwa drei Monaten. Das Ergebnis war eine statistisch signifikante Verbesserung der Lebendgeburtenraten nach dem Embryotransfer in der WS-TCM-Gruppe (61,3\%) im Vergleich zu ART allein (48,2\%) und zur Akupunkturgruppe $(50,8 \%)(p=0,03)$.

\section{Zusammenfassung der Studienergebnisse}

Die Datenlage zeigt einen deutlichen Trend zur Verbesserung verschiedener Parameter nach einer Behandlung mit chinesischer Medizin:

- Verbesserung der Schwangerschaftsraten pro Transfer nach Akupunktur um die Zeit des Transfers bei guter Embryoqualität,

- Verbesserung der Schwangerschaftsraten nach individualisierter Behandlung mit chinesischer Medizin über einen Zeitraum von drei bis sechs Monaten nach Embryotransfer,

- Verbesserung der kumulativen Schwangerschaftsraten ohne Maßnahmen der ART,

- Verbesserung der Lebendgeburtenraten.

\section{Integrative Kinderwunschbehandlung} mit chinesischer Medizin in der Praxis

Entsprechend der Diagnose in der CM werden vorbereitend auf eine künstliche Befruchtung, die im Zusammenhang mit dem Kinderwunsch relevanten Probleme und Pathomechanismen aus Sicht der $\mathrm{CM}$ behandelt. Mit Beginn der hormonellen Follikelstimulation der Eierstöcke setzt die CM ebenfalls einen entsprechenden Fokus: Die Eizellreifung wird unterstützt und die Gebärmutterschleimhaut vorbereitet für die Implantation des Embryos in die Gebärmutter. Dabei werden verschiedene Strategien verfolgt:

- Yin, Jing und Blut nähren, Qi und Yang stärken, Shen beruhigen 
- Dynamik und harmonischen Fluss von Qi und Blut gewährleisten

- Feuchtigkeit oder Schleim als mögliche Hinderungsursachen eliminieren

Die folgenden Behandlungsvorschläge sind die Essenz unserer eigenen klinischen Erfahrung und Praxis.

\section{Während der kontrollierten ovariellen Stimulation}

Die Akupunkturpunkte werden entsprechend der Therapiestrategie ausgewählt, einen Überblick gibt Tab. 1. Akupunkturpunkte, mit denen eine tonisierende Therapiestrategie verfolgt wird, werden tonisierend genadelt, während bei einer bewegenden Strategie eine sedierende bzw. zerstreuende Nadeltechnik angewendet wird und ein De Qi erreicht werden sollte. Mindestens ein lokaler Punkt im Unterbauch dient dazu, am Ort des Geschehens Qi und Blut zu bewegen. Pro Behandlung sollten nicht mehr als 10-12 Nadeln gestochen werden. Die konkrete Auswahl der Punkte kann entsprechend der weiteren Symptome und Zeichen der Patientin getroffen werden.

Die klassische Leitrezeptur während der ovariellen Stimulation ist Gui Shao Di Huang Tang (Liu Wei Di Huang Wan, modifiziert mit Dang Gui und Bai Shao zur Ernährung des Blutes) mit Wu Zi Yan Zong Wan. Diese beiden Leitrezepturen werden zusammen gegeben. Sie sind tonisierend und nährend, dabei ausgewogen, indem sie gleichzeitig pathologische Hitze abfließen lassen. Die Rezepturen werden durch Ergänzung einzelner Kräuter individuell angepasst. Einige Einzelkräuter, die in der Praxis häufig verwendet werden, sind beispielhaft in Tab. 1 aufgeführt. Kräuter, die bereits in den beiden genannten Rezepturen enthalten sind, werden in der Tabelle nicht noch einmal genannt.

Bei der Modifizierung muss das Temperaturverhalten der Kräuter beachtet werden (z.B. ist Dan Shen kühlend, während Chuan Xiong warm ist) ebenso wie die weiteren Eigenschaften der Kräuter. Wenn Feuchtigkeit oder Schleim vorhanden ist, sollte die Pathologie in der Vorbereitung auf die Stimulation behandelt werden. Die in Tab. 1 genannten Kräuter gehören nicht in die Kategorie der Feuchtigkeit transformierenden oder Schleim eliminierenden Kräuter, da es während der Stimulation selbst darum geht, die Qi- und Blut-Produktion sowie -Dynamik nicht zu stören und die Kollateralen zu öffnen.

\section{Um die Zeit des Embryotransfers}

Nach der ovariellen Stimulation werden die Follikel punktiert, außerhalb des Körpers mithilfe von IVF oder ICSI befruchtet und anschließend an Tag 2 bis 5 nach Punktion wieder in den Uterus der Frau transferiert (Embryotransfer). In der CM geht es unmittelbar vor und nach dem Transfer darum, den Uterus ruhig zu halten und den Shen zu beruhigen. Die Leitrezeptur ist Shao Yao Gan Cao Tang. Häufig verwendete Modifikationen sind Huang Qi und Shan Yao, um das Qi zu stärken und anzuheben, sowie ggf. Sheng Di Huang, um das

\begin{tabular}{|c|c|c|}
\hline Therapiestrategie & Akupunkturpunkte & CAT \\
\hline Blut, Yin und Jing tonisieren & & $\begin{array}{l}\text { Leitrezepturen während der Stimulation: } \\
\text { Gui Shao Di Huang Tang + Wu Zi Yan Zong Wan }\end{array}$ \\
\hline Qi tonisieren & Ma 36, BI 20, Mi 3, Ren 12 & Ren Shen, Dan Shen, Bai Zhu, Huang Qi, Tai Zi Shen \\
\hline Blut tonisieren & Ma 36, Mi 6 & E Jiao, Long Yan Rou \\
\hline Nieren-Yin nähren & Ni 6 , Ni 3, Ren 4 & Nu Zhen Zi, Han Lian Cao, Tian Men Dong \\
\hline Qi bewegen & Le 3 + Di 4, Gb 34, Gb 41, Gb 40 & Chai Hu, Xiang Fu \\
\hline Blut bewegen & Mi 10, Mi 8 & Dan Shen, Yu Jin, Ji Xue Teng, Chuan Xiong \\
\hline bei Feuchtigkeit/Schleim & Mi 9, Ma 40 & $\begin{array}{l}\text { Chen Pi, Mu Xiang, Yu Jin, Ji Xue Teng, Wang Bu Liu } \\
\text { Xing, Ze Lan }\end{array}$ \\
\hline lokal Qi und Blut bewegen & Ma 29, Zi Gong, Ren 4, Ren 6 & \\
\hline
\end{tabular}

Tab. 1

\section{Chinesische Arzneimittel (Leitrezeptur und Modifikationen) sowie Akupunktur- punkte entsprechend der Therapiestrategie}

\begin{tabular}{|c|c|c|}
\hline Therapiestrategie & Akupunkturpunkte & CAT \\
\hline $\begin{array}{l}\text { Nieren stabilisieren und Fötus beru- } \\
\text { higen }\end{array}$ & $\mathrm{Ni} 3, \mathrm{Ni} 6, \mathrm{Ni} 7$ & Leitrezeptur Shou Tai Wan \\
\hline Qi tonisieren (und anheben) & Ma 36, BI 20, Mi 3, Ren 12 & Shan Yao, Huang Qi \\
\hline Qi bewegen und harmonisieren & Le $3, \mathrm{~Gb} 41, \mathrm{~Gb} 40$ & - \\
\hline Shen beruhigen & Du 20, He 7, Du 24, Yin Tang, Pe 6 & - \\
\hline Blut kühlen & BI 17 & Sheng Di Huang \\
\hline
\end{tabular}

Tab. 2 


\begin{tabular}{l|l|l|}
\hline $\begin{array}{l}\text { Zusammenfassung der Rezepturen und Akupunkturpunkte begleitend } \\
\text { Zur künstlichen Befruchtung }\end{array}$ & CAT \\
\hline Zeitpunkt & Akupunkturpunkte & $\begin{array}{l}\text { Gui Shao Di Huang Tang + Wu Zi Yan Zong Wan } \\
\text { modifiziert }\end{array}$ \\
\hline $\begin{array}{l}\text { Beginn der Stimulation bis } 3 \text { Tage } \\
\text { vor Transfer }\end{array}$ & $\begin{array}{l}\text { Ma 36, Mi 6, Ni 3, Le 3, } \\
\text { Ma 29/Zi Gong und andere }\end{array}$ & Shao Yao Gan Cao Tang modifiziert \\
\hline $\begin{array}{l}\text { 2 Tage vor Transfer bis } 2 \text { Tage nach } \\
\text { Transfer }\end{array}$ & Delphi Consensus Protocol & Shou Tai Wan modifiziert \\
\hline $\begin{array}{l}\text { 3 Tage nach Transfer bis Schwanger- } \\
\text { schaftstest (bei positivem Test län- } \\
\text { ger) }\end{array}$ & Ma 36, Le 3, He 7, Du 20 u. a. \\
\hline
\end{tabular}

Tab. 3

\begin{tabular}{|c|c|c|}
\hline \multicolumn{3}{|c|}{$\begin{array}{l}\text { Überblick über die Indikationen für eine Behandlung mit chinesischer Medizin } \\
\text { und ihre Erfolgschancen }\end{array}$} \\
\hline $\begin{array}{l}\text { Problem aus Sicht der westli- } \\
\text { chen Medizin }\end{array}$ & $\begin{array}{l}\text { Wie sinnvoll ist eine zusätzliche Be- } \\
\text { handlung mit chinesischer Medizin? }\end{array}$ & Welche Probleme können dabei adressiert werden? \\
\hline idiopathische Infertilität & $* * * *$ & Verbesserung der reproduktiven Kompetenz \\
\hline Endometriose & $* * * *$ & $\begin{array}{l}\text { Verbesserung des Milieus und damit der Eizellqualität, } \\
\text { Verringerung der Schmerzen bis zur Erfüllung des Kinder- } \\
\text { wunschs }\end{array}$ \\
\hline habituelle Aborte & $* * *$ & $\begin{array}{l}\text { Verbesserung des Milieus, Verbesserung der Eizellqualität, } \\
\text { Verringerung der Abortraten }\end{array}$ \\
\hline polyzystisches Ovarialsyndrom & $* * *$ & $\begin{array}{l}\text { Verminderung der Abortraten } \\
\text { Verbesserung der Eizellreifung }\end{array}$ \\
\hline $\begin{array}{l}\text { Nidations-/ Implantationspro- } \\
\text { blem }\end{array}$ & $* *$ & Verbesserung der Schwangerschaftsraten \\
\hline $\begin{array}{l}\text { Oligo-Astheno-Teratozoosper- } \\
\text { mie-Syndrom }\end{array}$ & $* *$ & $\begin{array}{l}\text { Verbesserung aller Parameter des Spermiogramms für } \\
\text { eine Spontangravidität, Verbesserung der Spermienquali- } \\
\text { tät vor ART }\end{array}$ \\
\hline verminderte ovarielle Reserve & * & Verbesserung der Ovarialkapazität und der Eizellqualität \\
\hline
\end{tabular}

Tab. 4

Blut zu kühlen. Diese Rezeptur kann zwei Tage vor bis zwei Tage nach dem Transfer gegeben werden. Für die Akupunktur am Tag des Transfers empfiehlt sich das Delphi Consensus Protocol [15]:

- Vor dem Embryotransfer: Mi 8, Le 3, Ma 29, Mi 6

- Nach dem Embryotransfer: Ma 36, Mi 10, Ni 3, Du 20

Zusätzlich wird jeweils mindestens einer der folgenden Akupunkturpunkte genadelt: Pe 6, Yin Tang, He 7. Akupunktiert wird eine halbe Stunde vor und eine halbe Stunde nach dem Transfer, die Akupunkturnadeln bleiben jeweils $30 \mathrm{~min}$ in situ.

\section{Posttransfer}

Nach dem Transfer ist das Ziel der Behandlung das Qi zu tonisieren und anzuheben, alle Prozesse zu harmonisieren und den Shen zu beruhigen. Die am häufigsten verwendete klassische Rezeptur für diese Phase ist Shou Tai Wan, die Fötus-Langlebigkeits-Pille. Sie wird entsprechend der individuellen Zeichen und Symptome modifiziert, wie Tab. 2 beispielhaft aufführt.

Bei Auftreten von Krämpfen im Unterbauch kann posttransfer zusätzlich Shao Yao Gan Cao Tang gegeben werden. Im Verlauf des Prozesses kommt es unserer Erfahrung nach häufig zu Hitzesymptomen. In diesen Fällen geben wir Sheng Di Huang mit in die Rezep- tur. Um das Qi zu bewegen und zu harmonisieren sowie den Shen zu beruhigen ist die Akupunktur sehr gut geeignet. Eine Zugabe von chinesischen Arzneimitteln ist daher zur Umsetzung dieser Therapiestrategien nicht zusätzlich notwendig.

\section{Indikationen}

Welche Paare profitieren besonders von einer Behandlung mit chinesischer Medizin? Ein junges Paar mit Verschluss der Eileiter ist definitiv ein Fall für die ART, aber keiner für eine Behandlung mit chinesischer Medizin. Wann also sollten behandelnde Ärzte daran denken, betroffenen Paaren eine Therapie mit CM zu empfehlen? Tab. 4 zeigt einen Überblick über die typischsten Krankheitsbilder im Zusammenhang mit einem unerfüllten Kinderwunsch und gibt eine Einschätzung darüber, wie groß die Chancen mit CM sind, die Ergebnisse der ART zu verbessern sowie welche Probleme adressiert werden können. Sie basiert auf unseren Erfahrungen aus der klinischen Praxis.

\section{Hinweis}

Ein unerfüllter Kinderwunsch ist in aller Regel das Resultat verschiedener Probleme, die sich auf mehreren Ebenen abspielen. Auch 
in der chinesischen Medizin ist daher eine Spezialisierung aus unserer Sicht notwendig, um die Feinheiten erfassen und entsprechend behandeln zu können. Ärzte und Therapeuten, die keine Ausbildung in CAT haben, können problemlos die Akupunkturbehandlungen übernehmen, sollten aber für die Verschreibungen der Arzneimittel mit Therapeuten kooperieren, die eine fundierte Ausbildung in der chinesischen Arzneimitteltherapie haben und sich auf die integrative Kinderwunschbehandlung spezialisiert haben. Geeignete Therapeuten finden Sie z. B. unter tcm-kinderwunsch. info oder https://aborm.org.

Interessenkonflikt: Die Autoren erklären, dass keine wirtschaftlichen oder persönlichen Verbindungen bestehen.

\section{Online zu finden unter}

http://dx.doi.org/10.1055/a-0823-6215

\section{Literatur}

1 Anderson B, Haimovici F, Ginsburg E et al. In vitro fertilization and acupuncture: Clinical efficacy and mechanistic basis. Altern Ther Health Med 2007; 13 (3): $38-48$

$\overline{2}$ Blumenauer V, Czeromin U, Fehr D et al. D.I.R-Annual 2016 - The German IVF-Registry. J Reproduktionsmed Endokrinol 2017; 14 (6): 272-305
3 Vander Borght M, Wyns C. Fertility and infertility: Definition and epidemiology. Clinical Biochemistry 2018; 62: 2-10

4 Cao H, Han M, Ng EHY et al. Can Chinese herbal medicine improve outcomes of in vitro fertilization? A systematic review and meta-analysis of randomized controlled trials. PLoS ONE 2013; 8 (12): e81650

5 Dobos G, Tao I. The model of Western Integrative Medicine: The role of Chinese medicine. Chinese Journal of Integrative Medicine 2011; 17 (1): 11-20

6 El-Toukhy T, Sunkara SK, Khairy M et al. A systematic review and meta-analysis of acupuncture in in vitro fertilisation. BJOG: An International journal of Obstetrics and Gynaecology 2008; 115 (10): 1203-1213

7 Huang D, Huang G, Lu F et al. Acupuncture for infertility: Is it an effective therapy? Chinese Journal of Integrative Medicine 2011; 17 (5): 386-395

8 Hullender Rubin LE, Opsahl MS, Wiemer KE et al. Impact of whole systems traditional Chinese medicine on in-vitro fertilization outcomes. Reproductive BioMedicine Online 2015; 30 (6): 602-612

9 de Lacey SL, Sanderman E, Smith CA. Acupuncture in reproductive medicine: the motivations of infertile women to participate in a randomised controlled trial. J Psychosom Obstet Gynecol 2017; 39 (2): 112-120

$\overline{10}$ Manheimer $\mathrm{E}$, van der Windt $\mathrm{D}$, Cheng $\mathrm{K}$ et al. The effects of acupuncture on rates of clinical pregnancy among women undergoing in vitro fertilization: a systematic review and meta-analysis. Human reproduction update 2013; 19 (6): 696-713

\section{Rezepturen}

\section{Gui Shao Di Huang Tang}

Dang Gui (Angelicae sinensis Radix) $10 \mathrm{~g}$ - Blut ernährend und belebend

Bai Shao (Paeoniae Radix Alba) $10 \mathrm{~g}$ - Blut ernährend, Leber erweichend

Shu Di Huang (Rehmanniae Radix praeparata) $10 \mathrm{~g}$ - Blut ernährend

Shan Zhu Yu (Corni Fructus) $10 \mathrm{~g}$ - Essenz adstringierend, Blut und Yin stärkend

Shan Yao (Dioscoreae Rhizoma) $10 \mathrm{~g}$ - Qi der drei Erwärmer tonisierend

Fu Ling (Poria) $12 \mathrm{~g}$ - Qi stärkend und die unteren Wasserwege freihaltend

Mu Dan Pi (Moutan Cortex) $5 \mathrm{~g}$ - Hitze eliminierend und Blut kühlend

Ze Xie (Alismatis Rhizoma) $10 \mathrm{~g}$ - Harnfluss fördernd und Nässe ausleitend

\section{Wu Zi Yan Zong Wan}

Wu Wei Zi (Schisandrae Fructus) $12 \mathrm{~g}$ - Essenz adstringierend, Nieren stabilisierend

Fu Pen Zi (Rubi Fructus) $9 \mathrm{~g}$ - Nieren stabilisierend, Essenz adstringierend, Yang tonisierend

Tu Si Zi (Cuscutae Semen) $9 \mathrm{~g}$ - Nieren-Yin nährend und -Yang tonisierend, adstringierend

Che Qian Zi (Plantaginis Semen) $9 \mathrm{~g}$ - lässt Feuchtigkeit und Hitze abfließen, Harnfluss fördernd
Gou Qi Zi (Lycii Fructus) $12 \mathrm{~g}$ - Blut und Yin von Leber und Nieren nährend

\section{Shao Yao Gan Cao Tang}

Bai Shao (Paeoniae Radix Alba) $15 \mathrm{~g}$ - Yin und Blut nährend, zusammen mit Zhi Gan Cao entkrampfend

Zhi Gan Cao (Glycyrrhizae Radix praeparata) $15 \mathrm{~g}$ - Qi tonisierend

\section{Shou Tai Wan}

Tu Si Zi (Cuscutae Semen) $20 \mathrm{~g}$ - Nieren-Yin nährend und -Yang tonisierend, Fötus beruhigend

Sang ji Sheng (Taxilli Herba) $10 \mathrm{~g}$ - Nieren und Leber tonisierend, Yin und Blut nährend, Fötus beruhigend

Xu Duan (Dipsaci Radix) $10 \mathrm{~g}$ - Nieren und Leber tonisierend, sanft das Blut belebend, Fötus beruhigend

E Jiao (Asini Corii Colla) $10 \mathrm{~g}$ - Blut und Yin nährend, Blutungen stoppend

Die Rezepturen sind folgenden Standardwerken entnommen: Bensky D, Barolet R. Chinesische Arzneimittelrezepte und Behandlungsstrategien. Bad Kötzting: Verlag für Ganzheitliche Medizin; 1996

Ehling D. Handbuch Chinesische Kräuterrezepte. München: Urban \& Fischer; 2001

Die Mengenangaben der Einzelkräuter stammen von den Autoren dieses Artikels. 
$\overline{11}$ Paulus WE, Zhang M, Strehler E et al. Influence of acupuncture on the pregnancy rate in patients who undergo assisted reproduction therapy. Fertility and Sterility 2002; 77 (4): 721-724

$\overline{12}$ Qian Y, Xia XR, Ochin $\mathrm{H}$ et al. Therapeutic effect of acupuncture on the outcomes of in vitro fertilization: a systematic review and meta-analysis. Arch Gynecol Obstet 2017; 295 (3): 543-558

$\overline{13}$ Qu F, Zhou J, Ren RX. Effects of acupuncture on the outcomes of in vitro fertilization: A systematic review and meta-analysis. J Altern Complement Med 2012; 18 (5): 429-439

$\overline{14}$ Ried K. Chinese herbal medicine for female infertility: An updated meta-analysis. Complementary Therapies in Medicine 2015; 23 (1): 116-128

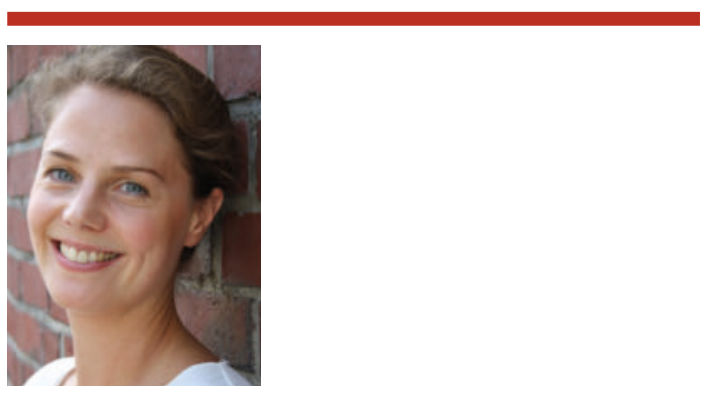

Nora Giese

Praxis für Chinesische Medizin

Kaiserstraße 237, 53113 Bonn

info@praxis-giese.com

www.praxis-giese.com

Nora Giese ist Heilpraktikerin und Fellow of ABORM. Sie arbeitet mit dem Schwerpunkt Gynäkologie und Integrative Kinderwunschbehandlung in ihrer Praxis in Bonn. Ihre Zusatzqualifikation erwarb sie bei der Deutschen Gesellschaft für Reproduktionsmedizin (DGRM). Sie unterrichtet Chinesische Medizin u.a. an der Universität Witten-Herdecke und leitet eine Ausbildung für Integrative Kinderwunschbehandlung mit chinesischer Medizin. Publikation 2018: „Endometriose-Ratgeber Traditionelle Chinesische Medizin: Endometriose mit Chinesischer Medizin behandeln“ im Verlag Müller \& Steinicke. $\overline{15}$ Smith CA, Grant S, Lyttleton J et al. Using a Delphi consensus process to develop an acupuncture treatment protocol by consensus for women undergoing Assisted Reproductive Technology (ART) treatment. BMC Complement Altern Med 2012; 12 (1): 1055

$\overline{16}$ Ying CC, Dix S, Ng EHY et al. Acupuncture and assisted reproductive technology. Cochrane Database of Systematic Reviews 2013; 26 (7)

$\overline{17}$ Zegers-Hochschild F, Adamson GD, de Mouzon J et al. International Committee for Monitoring Assisted Reproductive Technology (ICMART) and the World Health Organization (WHO) revised glossary of ART terminology. Fertility and Sterility 2009; 92 (5): 1520-1524

$\overline{18}$ Zheng $\mathrm{CH}$, Huang $\mathrm{GY}$, Zhang MM et al. Effects of acupuncture on pregnancy rates in women undergoing in vitro fertilization: A systematic review and meta-analysis. Fertility and Sterility 2012; 2: 599-611

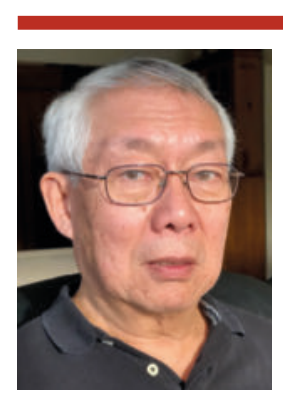

\section{Fransiscus Sulistyo-Winarto} praxis@sulistyo.de

Fransiscus Sulistyo-Winarto ist Facharzt für Gynäkologie und Geburtshilfe, sowie Facharzt für Allgemeinmedizin und arbeitet als Arzt in eigener Praxis in Dormagen. Er ist Lehrbeauftragter der Universität Witten-Herdecke für Chinesische Medizin und hält international Vorträge zur Chinesischen Medizin. Er ist Mitglied der Deutschen Gesellschaft für Reproduktionsmedizin (DGRM) und Gründungsmitglied des Europäischen Forum der Integrativen Chinesischen Medizin und Reproduktionsmedizin in Bonn. 